\title{
Bananas and People in the Homeland of Genus Musa: Not just pretty fruit \\ Jean Kennedy
}

\section{Research}

\begin{abstract}
Bananas, perhaps the most recognizable of fruits, are nowhere more genetically diverse than in the southwest $\mathrm{Pa}$ cific, where parthenocarpic fruit originated according to recent biomolecular evidence. In the wider Indo-Malesian area, homeland of the genus Musa L., understanding the domestication of bananas must include consideration of a much greater range of Musa species than just the few implicated in the parentage of the modern cultivars with seedless fruit.
\end{abstract}

Despite ethnobotanical evidence that the genus has been valued for many more products than the edible fruit, the role of other products in the process of domestication is seldom considered. As well as documenting the development and spread of seedless cultivars, we need to develop models of the fundamental Indo-Malesian practices through which the greater range of species and products, seeds and all, were managed in diverse socio-cultural and environmental circumstances.

\section{Introduction}

The natural range of the genus Musa L. extends throughout the Indo-Malesian region, in tropical and subtropical areas from Sri Lanka and eastern India, across south China and Southeast Asia to the southwest Pacific and northern Australia (Figure 1). There are more than 50 Musa species (Constantine 1999-2008, Häkkinen \& Väre 2008), most of which are large perennial tropical herbs, mainly of lowland areas with high temperature and humidity.

The edibility of traditional and modern fruit cultivars traces back to parthenocarpic forms that arose in one or two subspecies of Musa acuminata Colla in the southwest Pacific, at the eastern extremity of the range of that species and of the genus (Carreel et al. 2002, Perrier et al. 2009). Parthenocarpy in bananas entails the development of ed- ible pulp without pollination; it does not guarantee seedlessness, which depends on additional mechanisms that are at least partly independent of parthenocarpy (Argent 1979, Simmonds 1959: 28-9, 1962). As vegetatively propagated parthenocarpic land races spread west through the area of other Musa species, they were involved in complex hybridizations that produced many new land races with virtually seedless fruit (see Perrier et al. 2009). While some of these have been transmitted to the rest of the tropical and subtropical world, Malesia retains the highest diversity of land races, and nowhere are these more diverse than in Papua New Guinea (see Kennedy 2008, n.d. for further discussion and references).

Understandably, a great deal of attention has been given to explicating the genetics and biogeography of the process by which bananas with seedless fruit were domesticated (Kennedy 2008, n.d.; Perrier et al. 2009, Simmonds 1962). My argument here elaborates on points I (and others) have previously made only in passing: we need to recontextualize understanding of the domestication of bananas within the frameworks of historical ecology for the Malesian region that are emerging from multidisci-

\section{Correspondence}

Jean Kennedy, Department of Archaeology and Natural History, Research School of Pacific and Asian Studies, Australian National University, Canberra ACT 0200, AUSTRALIA.

jean.kennedy@anu.edu.au

Ethnobotany Research \& Applications 7:179-197 (2009)

Published: July 30, 2009 


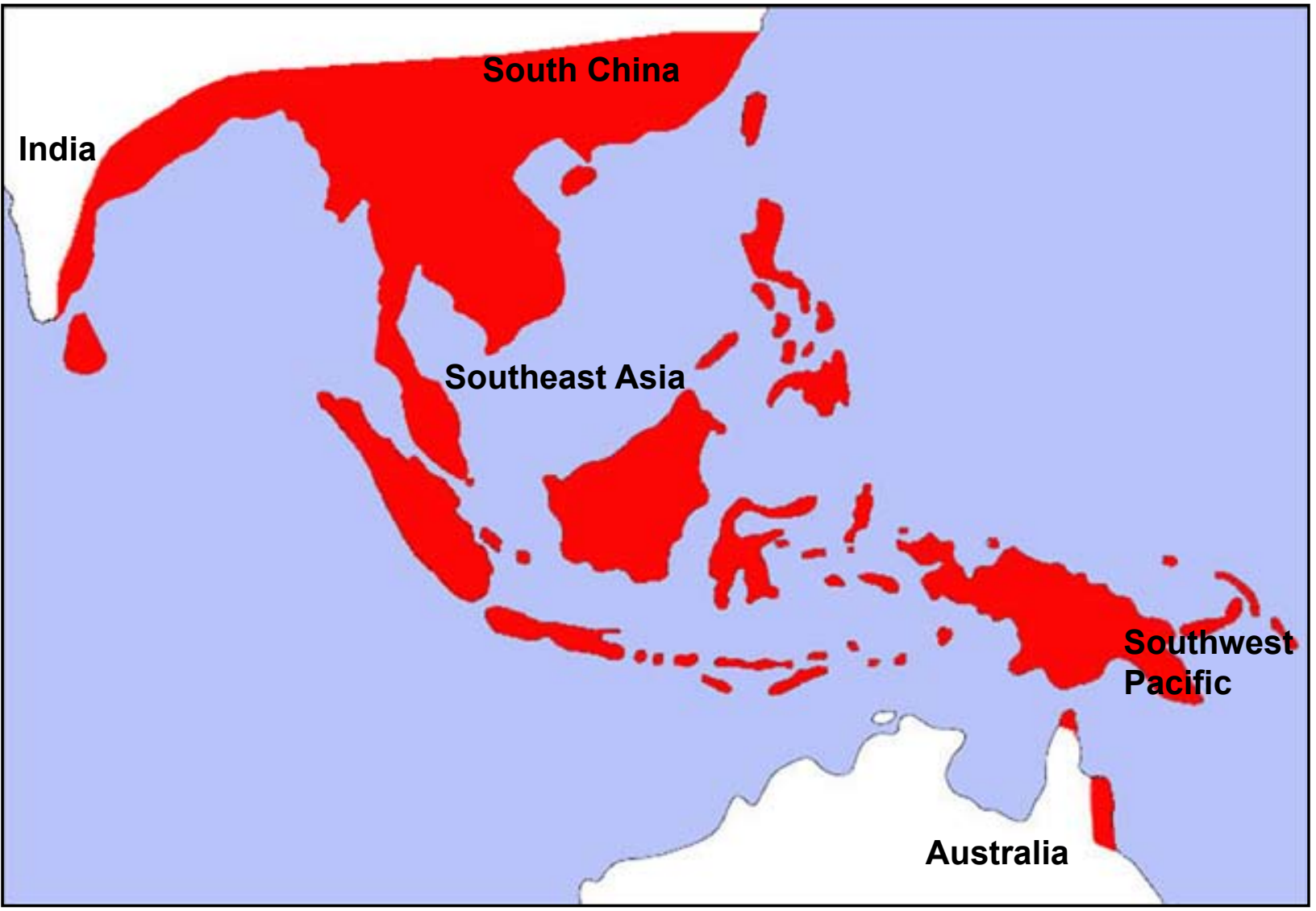

Figure 1. Distribution of genus Musa L. in the Indo-Malesian region.

plinary studies of environmental and cultural changes in the late Pleistocene and Holocene, and to take a broader view of banana domestication than is usual. Documenting the development of the familiar edible bananas of today has been the main focus of studies of the interactions of humans and bananas, and this focus is still reflected in most current archaeobotanical discussion. But the utility of a phylogenetic outline of the development of edible bananas for the project of improved breeding does not necessarily give it power to guide prehistoric research. We need to know more about bananas than how the fruits were made succulent and seedless.

The basic proposition of this paper is that the Musaceae as a whole, like 'cassowaries and kiwis, and big bouquets of roses' (Hays 1991:113), have high ethnobiological salience, independent of forms with edible fruit. In support of this proposition, I explore examples of the very wide range of uses documented throughout Indo-Malesia. Given that such uses are widespread and probably reflect ancient practices, it is likely that human selective pressures have affected other aspects of the plants besides the edibility of fruit. If this is correct, then the tendency to equate seediness with wildness is very misleading. In my hypothetical encounters of early humans and bananas, seediness did not matter, and the fruit was probably not the main focus of attention. To assume otherwise is teleological.
It follows that the vegetatively propagated parthenocarpic bananas from the eastern edge of Malesia spread west into a world in which other Musa species had already been subject to the manipulations of humans, for uses including medicine, fiber, wrapping material and ritual, as well as food. All such uses entail the possibility of selective pressures that have little or nothing to do with edibility of the fruit. These humanly modified plant populations are termed 'cultiwild' by De Langhe et al. (2009). As we develop better archaeobotanical tools for investigating the prehistoric world of tropical plants, we should keep these possibilities in mind.

\section{Ecology and Ethnobotany of Indo-Malesian Musaceae}

Simmonds (1962:34) described wild Musa species as 'jungle weeds' of disturbed habitats, such as talus slopes, unstable hillsides or forest gaps. Disturbance is an integral part of the dynamic growth cycle of the rainforest, providing niches for understory plants such as Musaceae (Argent 1979, Whitmore 1984). When Homo sapiens entered the world of wild Musa species c. 60,000 years ago, the niches of these plants and people must have overlapped substantially. A growing body of archaeological evidence shows Pleistocene human presence in forested environ- 


\section{Kennedy - Bananas and People in the Homeland of Genus Musa: Not just pretty fruit}

ments of Island Southeast Asian and the New Guinea region (Barker 2005, Barker et al. 2005, Barker et al. 2007, Kealhofer 2003, Latinis 2000, Mercader 2003, Pavlides \& Gosden 1994). It is highly likely that early humans were especially drawn to forest gaps and may have increased their extent (Groube 1989). The stately and often spectacular Musaceae are far too conspicuous to have been overlooked (Figures 2 and 3 ). Thus began a long period of interaction with humans that continues to the present.

It is well known that Musaceae are very useful. Leaves and inflorescences are prominent in many Southeast Asian food markets (Figures 4 and 5), and increasingly in metropolitan markets of the First World. The multiplicity of uses is manifest in compilations of tropical products, such as the invaluable colonial works of Burkill (1935), Heyne (1950), Ochse (1977) and Watt (1972), as well as in more specialist literature on botanical and agronomic aspects of bananas, such as Purseglove (1975), Simmonds (1959 and 1962) and publications of the International Network for the Improvement of Banana and Plantain (INIBAP) (e.g., Sharrock 1996).

All parts of the plant are useful, including seedy fruit, inflorescence, leaf, pseudostem, corm and rhizome; they furnish food, fodder, medicine, domestic materials and shelter. Plants also have ritual and ceremonial significance. Table 1 lists miscellaneous uses, other than consump-

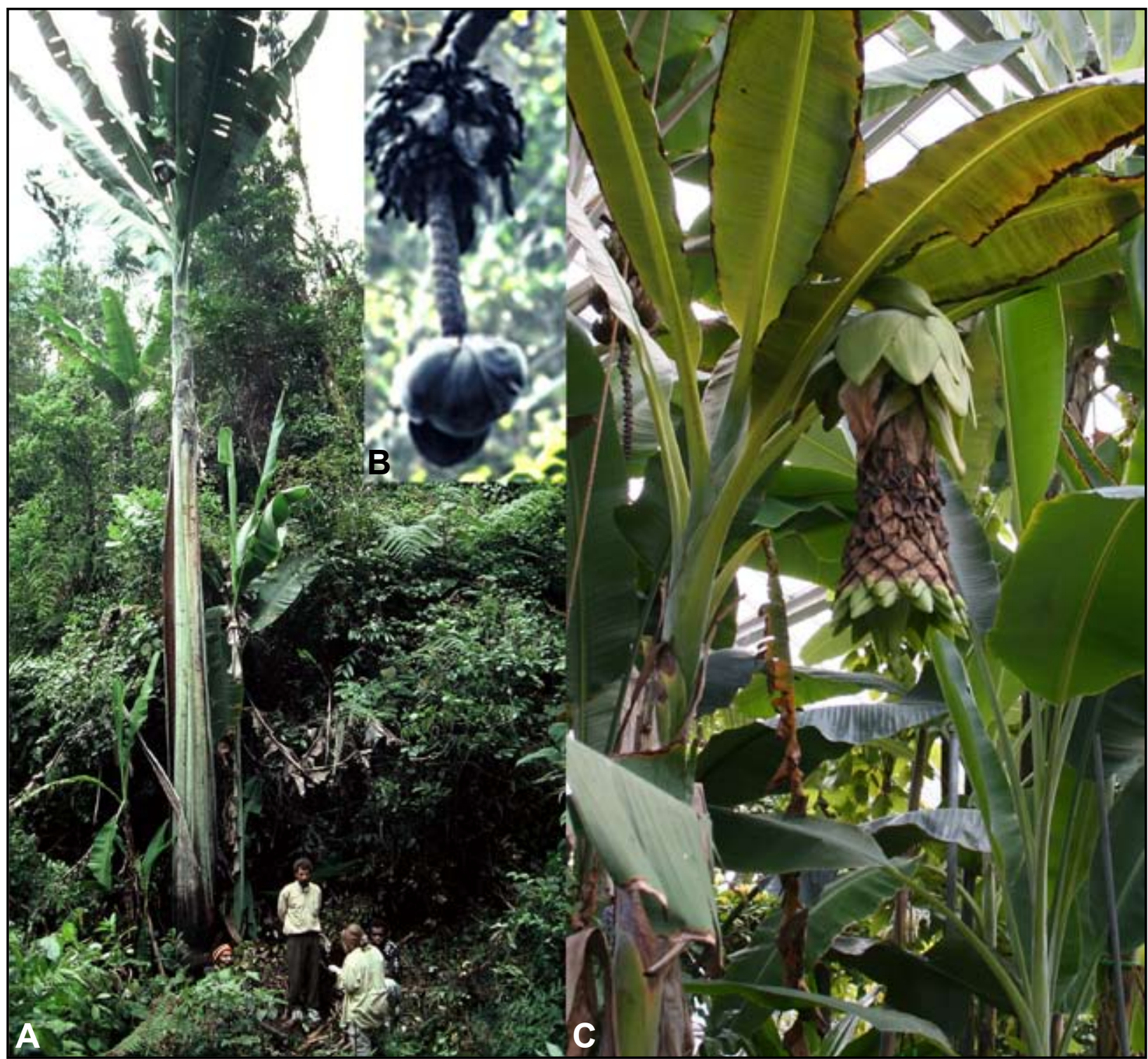

Figure 2. Many Musa species grow to statuesque proportions and dominate the landscape. A, B. The largest is Musa ingens Simmonds, a New Guinea Highlands endemic; C. Ensete glaucum (Roxb.) Cheesman has a wide but scattered distribution from the Eastern Himalayan fringe to New Guinea. (Photos A, B. by Jeff Daniells, Queensland DPI; C. by Shu Suehiro, Kamisu-shi, Japan.) 


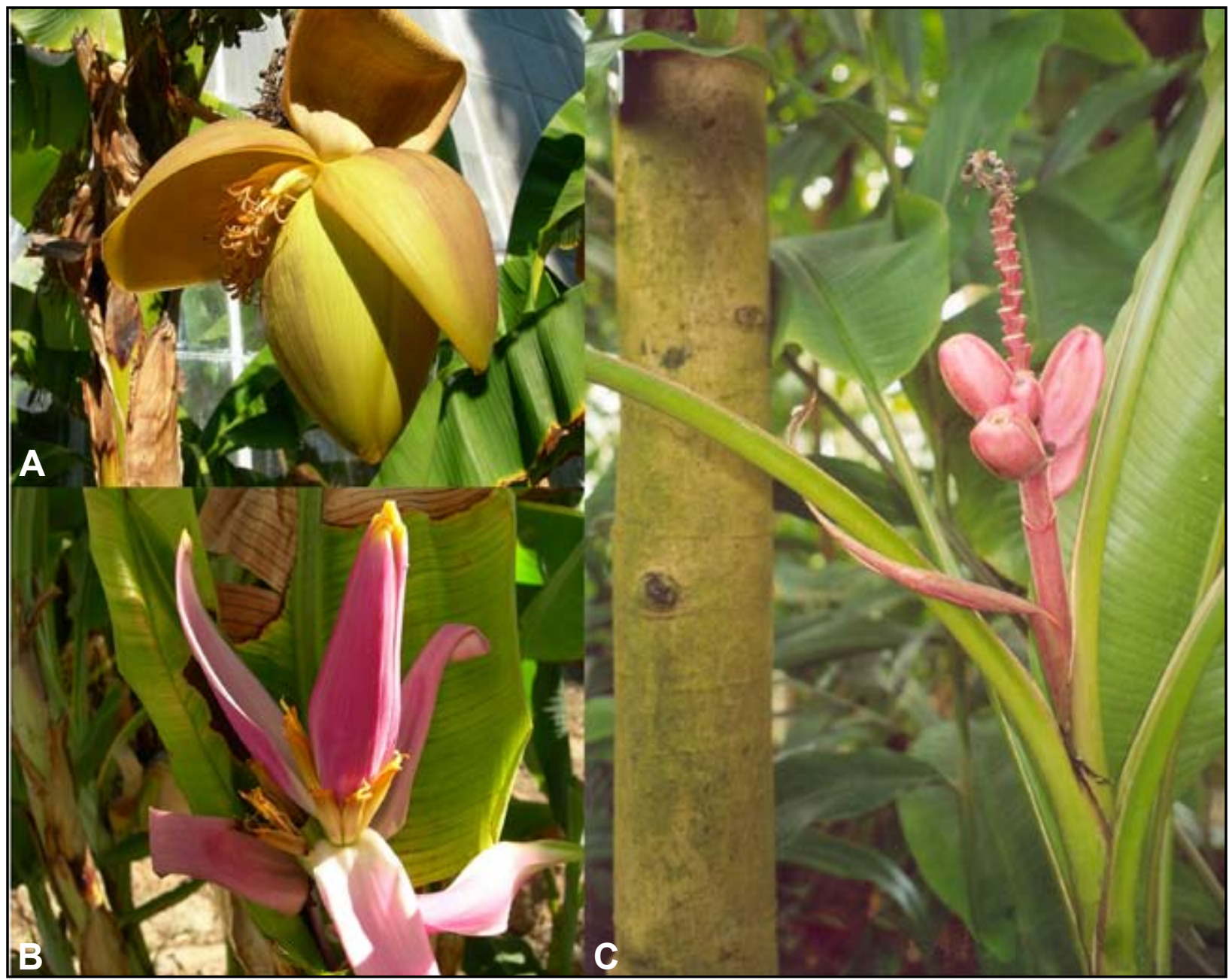

Figure 3. The flowers and fruit of many Musa species are conspicuous and appeal to horticulturally-inclined humans as well as to birds and bats: A. Musa basjoo von Siebold ex Y. linuma, University of California Botanical Garden, Berkeley; B. Musa ornata Roxb., local nursery, rural central Luzon, Philippines; C. Musa velutina H. Wendl. \& Drude, Royal Botanical Gardens, Kew. Photos by the author.

Figure 4 (right). In Thai food markets, many parts of Musaceae species are represented: A. Inner core of Musa sp pseudostem is eaten cooked, Chiang Mai; B. Terminal inflorescences are eaten cooked or raw, Chiang Mai; C. Immature fruit of Musa balbisiana Colla eaten as salad, Nong Khai; D. Ripe fruit of Ensete glaucum (Roxb.) Cheesman, also eaten raw, Udon Thani. Photos by Gianni Bertossa and Christiane Jacquat, Institute of Plant Biology \& Department of Pre- and Protohistory, University of Zurich.

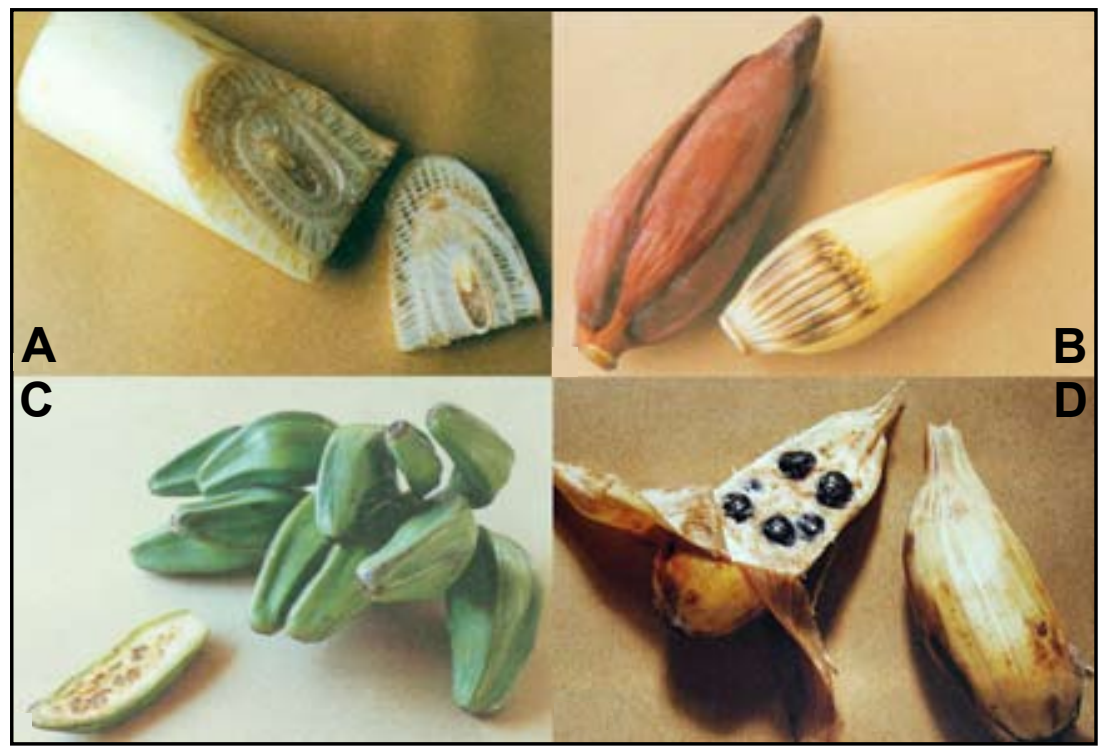




\section{Kennedy - Bananas and People in the Homeland of Genus Musa: Not just pretty fruit}

tion of seedless fruit, of Indo-Malesian Musaceae. Given the labyrinthine complexity of literary genres which might refer to bananas, I make no claim that this list is comprehensive in terms of uses or references; it is intended to be exemplary. I have revised the nomenclature, where possible, using the extensive cross-referencing of species names compiled by Constantine (1999-2008). For the sake of brevity, I have somewhat arbitrarily excluded African uses of Musaceae (genus Ensete Horan. is native there, but not genus Musa), while Polynesia and Micronesia are included because their cultural connec-

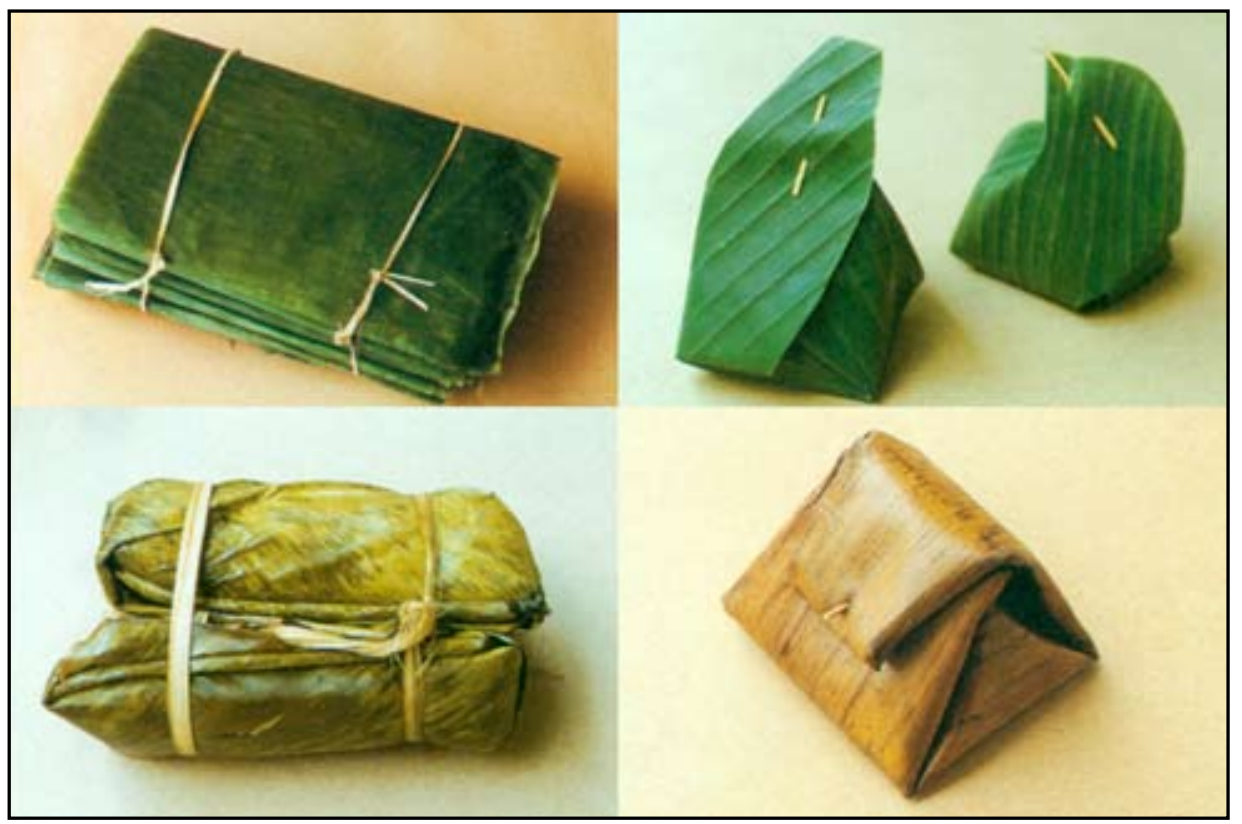

Figure 5. Leaves are widely used for packaging, especially for food, as in these examples of Thai takeaways. Photos by Gianni Bertossa and Christiane Jacquat, Institute of Plant Biology \& Department of Pre- and Protohistory, University of Zurich.

Table 1. Miscellaneous uses of banana plants (family Musaceae) in the Indo-Malesian region.

\begin{tabular}{|c|c|c|c|c|}
\hline \multicolumn{5}{|l|}{ Category } \\
\hline Part used & Use & Genus/species & Location(s) & Citations \\
\hline \multicolumn{5}{|l|}{ Food } \\
\hline $\begin{array}{l}\text { terminal } \\
\text { inflorescence/ } \\
\text { male bud }\end{array}$ & salad/cooked & $\begin{array}{l}\text { many spp/cvs, especially } \\
\text { Musa balbisiana Colla, } \\
\text { also Ensete glaucum } \\
\text { (Roxb.) Cheesman, } \\
\text { Musa acuminata ssp } \\
\text { microcarpa (Becc.) } \\
\text { Simmonds and var } \\
\text { zebrina (Van Houtte ex } \\
\text { Planch.) R.E.Nasution, } \\
\text { Musa campestris Becc., } \\
\text { Musa hirta Becc., Musa } \\
\text { itinerans Cheesman, } \\
\text { Musa lawitiensis } \\
\text { Nasution \& Supard., } \\
\text { Musa nagensium Prain, } \\
\text { Musa ornata Roxb., } \\
\text { Musa peekelii Lauterb., } \\
\text { Musa salaccensis } \\
\text { Zoll., Musa velutina } \\
\text { H. Wendl. \& Drude, } \\
\text { Musa violascens Ridl. }\end{array}$ & widespread & $\begin{array}{l}\text { Arnaud \& Horry 1997, Burkill } \\
\text { 1935, Christensen 2002, } \\
\text { Dassanayake \& Clayton 2000, } \\
\text { De Langhe et al. 2000, Heyne } \\
\text { 1950, Jacquat 1990, Lentfer } \\
\text { 2003, Noweg et al. 2003, } \\
\text { Ochse 1977, Rumphius 1747, } \\
\text { Simmonds 1956, 1959, Uma } \\
\text { 2006, Uphof 1968, Watt } 1972\end{array}$ \\
\hline male flowers & cooked & edible hybrid cvs & PNG & Argent 1979 \\
\hline $\begin{array}{l}\text { nectar from } \\
\text { male flowers }\end{array}$ & baby-food & Pacific plantain cvs & Hawaii & Nelson et al. 2006 \\
\hline $\begin{array}{l}\text { dry } \\
\text { inflorescence }\end{array}$ & salt & Musa sp & PNG & Petir et al. 1998 \\
\hline
\end{tabular}




\begin{tabular}{|c|c|c|c|c|}
\hline \multicolumn{5}{|l|}{ Category } \\
\hline Part used & Use & Genus/species & Location(s) & Citations \\
\hline \multirow[t]{2}{*}{ seedy fruit } & raw/cooked & $\begin{array}{l}\text { many spp, including } E . \\
\text { glaucum, M. acuminata } \\
\text { ssp, Musa aurantiaca } \\
\text { G. Manner ex Baker, } M \text {. } \\
\text { balbisiana, Musa maclayi } \\
\text { F. Muell. ex Mikl.-Maclay } \\
\text { M. peekelii, Musa } \\
\text { sanguinea Hook.f., } M \text {. } \\
\text { velutina, } M \text {. violascens }\end{array}$ & widespread & $\begin{array}{l}\text { Anderson 1993, Burkill 1935, } \\
\text { Häkkinen 2006, Jacquat 1990, } \\
\text { Lentfer 2003, Nguyen 2006, } \\
\text { Noweg et al. 2003, Ochse } \\
\text { 1977, Rumphius 1747, Uma } \\
\text { 2006, Yen \& Gutierrez } 1976\end{array}$ \\
\hline & $\begin{array}{l}\text { raw, sieved pulp } \\
\text { as baby food }\end{array}$ & M. balbisiana & N.E. India & Uma 2006 \\
\hline fruit peels & cooked & edible cvs & Java & Ochse 1977 \\
\hline $\begin{array}{l}\text { immature } \\
\text { seedy fruit }\end{array}$ & $\begin{array}{l}\text { raw, cooked, } \\
\text { pickled }\end{array}$ & $\begin{array}{l}\text { many spp, including } M \text {. } \\
\text { balbisiana, } M \text {. lawitiensis }\end{array}$ & widespread & $\begin{array}{l}\text { Christensen 2002, Jacquat } \\
\text { 1990, Nguyen 2006, Ochse } \\
\text { 1977, Simmonds } 1956 \\
\text { \& 1959, Uphof } 1968\end{array}$ \\
\hline pseudostem & $\begin{array}{l}\text { cooked, processed } \\
\text { for starch, } \\
\text { famine food }\end{array}$ & $\begin{array}{l}\text { many spp/cvs, including } \\
\text { M. balbisiana, Musa } \\
\text { flaviflora Simmonds, } \\
\text { Musa ingens Simmonds } \\
\text { M. itinerans, Musella } \\
\text { lasiocarpa (Franch.) } \\
\text { C.Y. Wu ex Li } \\
\end{array}$ & widespread & $\begin{array}{l}\text { Burkill 1935, De Langhe et al. } \\
\text { 2000, Hendrickx 2007, Heyne } \\
\text { 1950, Jacquat 1990, Lentfer } \\
\text { 2003, Liu et al. 2003, Ochse } \\
\text { 1977, Powell 1976, Rumphius } \\
\text { 1747, Sillitoe 1983, Simmonds } \\
\text { 1959, Uma 2006, Watt } 1972 \\
\end{array}$ \\
\hline $\begin{array}{l}\text { young leaf- } \\
\text { sheath }\end{array}$ & cooked & E. glaucum & Java & Ochse 1977 \\
\hline sucker/shoot & cooked & $\begin{array}{l}\text { many spp/cvs, including } \\
\text { M. acuminata ssp } \\
\text { microcarpa and ssp } \\
\text { zebrina, M. balbisiana, } \\
\text { Musa beccarii Simmonds, } \\
\text { M. campestris, } M . \\
\text { hirta, M. lawitiensis, } M \text {. } \\
\text { violascens, } M \text {. lasiocarpa } \\
\end{array}$ & $\begin{array}{l}\text { Borneo, } \\
\text { N E India, } \\
\text { Yunnan }\end{array}$ & $\begin{array}{l}\text { Christensen 2002, Häkkinen 2006, } \\
\text { Noweg et al. 2003, Ochse 1977, } \\
\text { Simmonds 1959, Uma } 2006\end{array}$ \\
\hline corm/rhizome & cooked & $\begin{array}{l}\text { many spp/cvs, including } \\
\text { M. acuminata ssp } \\
\text { zebrina, M. balbisiana }\end{array}$ & widespread & $\begin{array}{l}\text { Bailey } 1900 \text {, Burkill 1935, } \\
\text { Massal \& Barrau 1956, Mogina } \\
\text { 2002, Ochse 1977, Rumphius } \\
\text { 1747, Simmonds } 1959\end{array}$ \\
\hline $\begin{array}{l}\text { ash of whole } \\
\text { plant }\end{array}$ & seasoning & edible Musa cvs & India & Watt 1972 \\
\hline \multicolumn{5}{|l|}{ Fodder } \\
\hline whole plant & pig food & M. lasiocarpa & Yunnan & Liu et al. 2003, Long et al. 2008 \\
\hline \multirow[t]{2}{*}{$\begin{array}{l}\text { pseudostem, } \\
\text { stalk of } \\
\text { inflorescence } \\
\text { and fruit, leaf, } \\
\text { rhizome }\end{array}$} & pig and cattle food & $\begin{array}{l}\text { edible cvs, E. glaucum, } \\
\text { M. acuminata, M. } \\
\text { balbisiana, M. itinerans, } \\
\text { Musa yunnanensis } \\
\text { Häkkinen \& Wang }\end{array}$ & widespread & $\begin{array}{l}\text { Anderson 1993, Burkill 1935, De } \\
\text { Langhe et al. 2000, Häkkinen \& } \\
\text { Wang 2007, Häkkinen et al. 2008, } \\
\text { Heyne 1950, Lentfer 2003, Nelson } \\
\text { et al. 2006, Rumphius 1747, } \\
\text { Simmonds 1959, Uphof 1968, } \\
\text { Watt 1972, Wu \& Kress } 2000\end{array}$ \\
\hline & $\begin{array}{l}\text { food for captive } \\
\text { cassowaries }\end{array}$ & edible Musa cvs & $\begin{array}{l}\text { PNG } \\
\text { Highlands }\end{array}$ & Sterly 1997 \\
\hline
\end{tabular}


Kennedy - Bananas and People in the Homeland of Genus Musa:
Not just pretty fruit

\begin{tabular}{|c|c|c|c|c|}
\hline \multicolumn{5}{|l|}{ Category } \\
\hline Part used & Use & Genus/species & Location(s) & Citations \\
\hline $\begin{array}{l}\text { outer leaf- } \\
\text { sheath }\end{array}$ & elephant food & edible Musa cvs & India & Watt 1972 \\
\hline \multirow[t]{2}{*}{ fruit } & $\begin{array}{l}\text { food for captive } \\
\text { cassowaries }\end{array}$ & M. ingens & $\begin{array}{l}\text { PNG } \\
\text { Highlands }\end{array}$ & Sterly 1997 \\
\hline & food for piglets & M. nagensium & N E India & Uma 2006 \\
\hline corm/rhizome & cattle food & $\begin{array}{l}\text { M. acuminata, } M . \\
\text { aurantiaca, } \text { M. balbisiana, } \\
\text { Musa laterita Cheesman, } \\
\text { M. nagensium, M. ornata }\end{array}$ & N E India & Uma 2006 \\
\hline \multicolumn{5}{|l|}{ Medicine } \\
\hline $\begin{array}{l}\text { juice/sap of } \\
\text { pseudostem, } \\
\text { stalk of } \\
\text { inflorescence, } \\
\text { roots and } \\
\text { flowers }\end{array}$ & $\begin{array}{l}\text { astringent, styptic, } \\
\text { mouthwash, gargle, } \\
\text { antipyretic, wound } \\
\text { dressing, burns, } \\
\text { tonic, internal } \\
\text { complaints, } \\
\text { treatment/antidote } \\
\text { for diabetes, } \\
\text { gonorrhoea, kidney } \\
\text { inflammation, } \\
\text { mercury poisoning, } \\
\text { drunkenness, } \\
\text { abortifacient, } \\
\text { hair tonic }\end{array}$ & $\begin{array}{l}\text { many spp/cvs, } \\
\text { including E. glaucum, } \\
\text { M. balbisiana, Musa } \\
\text { basjoo von Siebold ex Y. } \\
\text { linuma, M. nagensium, } \\
\text { M. lasiocarpa }\end{array}$ & widespread & $\begin{array}{l}\text { Burkill 1935, Christensen } 2002 \text {, } \\
\text { Hendrickx 2007, Heyne 1950, } \\
\text { Jacquat 1990, Liu et al. 2003, } \\
\text { Nelson et al. 2006, Perry 1980, } \\
\text { Powell 1976, Reynolds \& Fang } \\
\text { 1940, Rumphius 1747, Sterly } \\
\text { 1997, Uma 2006, Uphof 1968, } \\
\text { Watt 1972, Whistler } 1992\end{array}$ \\
\hline $\begin{array}{l}\text { decoctions/ } \\
\text { infusions } \\
\text { of leaves, } \\
\text { flowers, roots, } \\
\text { pseudostem }\end{array}$ & $\begin{array}{l}\text { various internal } \\
\text { complaints, internal } \\
\text { hemorrhage, } \\
\text { burns, swellings }\end{array}$ & $\begin{array}{l}\text { many spp/cvs, including } \\
\text { Musella lasiocarpa }\end{array}$ & widespread & $\begin{array}{l}\text { Anderson 1993, Burkill 1935, } \\
\text { Heyne 1950, Jacquat 1990, Liu } \\
\text { et al. 2003, Nelson et al. 2006, } \\
\text { Reynolds \& Fang 1940, Rumphius } \\
\text { 1747, Watt 1972, WHO } 1998\end{array}$ \\
\hline root & $\begin{array}{l}\text { ayurvedic } \\
\text { preparations }\end{array}$ & $\begin{array}{l}\text { M. acuminata, } M \text {. } \\
\text { aurantiaca, M. balbisiana, } \\
\text { M. laterita, } M . \text { nagensium, } \\
\text { M. ornata }\end{array}$ & N E India & Uma 2006 \\
\hline \multirow{2}{*}{$\begin{array}{l}\text { skin of } \\
\text { pseudostem }\end{array}$} & insect bites & edible cvs & PNG & Powell 1976 \\
\hline & $\begin{array}{l}\text { tonic for humans } \\
\text { and pigs }\end{array}$ & M. ingens & $\begin{array}{l}\text { PNG } \\
\text { Highlands }\end{array}$ & Sterly 1997 \\
\hline $\begin{array}{l}\text { fruit pulp and } \\
\text { pounded skin }\end{array}$ & poultice & edible Musa cvs & $\begin{array}{l}\text { Malaysia, } \\
\text { PNG, } \\
\text { Polynesia, } \\
\text { Thailand }\end{array}$ & $\begin{array}{l}\text { Burkill 1935, Jacquat 1990, } \\
\text { Nelson et al. 2006, Sterly } \\
\text { 1997, Whistler } 1992\end{array}$ \\
\hline ripe fruit & $\begin{array}{l}\text { diarrhoea, } \\
\text { dysentery }\end{array}$ & edible Musa cvs & India & Watt 1972 \\
\hline $\begin{array}{l}\text { seedy } \\
\text { fruit, ripe }\end{array}$ & $\begin{array}{l}\text { diarrhoea, } \\
\text { dysentery }\end{array}$ & Musa sp & Mindanao & Dampier 1927 \\
\hline roasted fruit & $\begin{array}{l}\text { diarrhoea, } \\
\text { dysentery }\end{array}$ & edible Musa cvs & $\begin{array}{l}\text { Indonesia, } \\
\text { PNG } \\
\text { Highlands }\end{array}$ & Heyne 1950, Sterly 1997 \\
\hline $\begin{array}{l}\text { immature } \\
\text { seedy fruit, } \\
\text { roasted }\end{array}$ & $\begin{array}{l}\text { diarrhoea, } \\
\text { dysentery }\end{array}$ & M. balbisiana & Indonesia & Heyne 1950 \\
\hline
\end{tabular}




\begin{tabular}{|c|c|c|c|c|}
\hline \multicolumn{5}{|l|}{ Category } \\
\hline Part used & Use & Genus/species & Location(s) & Citations \\
\hline fruit skin & $\begin{array}{l}\text { tincture for internal } \\
\text { complaints }\end{array}$ & M. balbisiana & Cambodia & Kham 2004 \\
\hline $\begin{array}{l}\text { young flower } \\
\text { buds }\end{array}$ & $\begin{array}{l}\text { respiratory } \\
\text { complaints }\end{array}$ & Musa sp & $\begin{array}{l}\text { PNG } \\
\text { Highlands }\end{array}$ & Powell 1976 \\
\hline \multirow[t]{3}{*}{ leaves } & dressings & $\begin{array}{l}\text { many spp/cvs, } \\
\text { including } M . \text { aurantiaca, } \\
\text { M. balbisiana, } M \text {. } \\
\text { nagensium, } M \text {. ornata }\end{array}$ & widespread & $\begin{array}{l}\text { Burkill 1935, Perry 1980, Sterly } \\
\text { 1997, Uma 2006, Watt } 1972\end{array}$ \\
\hline & elephant medicine & $\begin{array}{l}\text { Musa acuminata } \\
\text { ssp malaccensis } \\
\text { (Ridl.) Simmonds }\end{array}$ & $\begin{array}{l}\text { Malaysia, } \\
\text { Indonesia }\end{array}$ & Burkill 1935 \\
\hline & $\begin{array}{l}\text { eaten as } \\
\text { abortifacient }\end{array}$ & Musa spp & PNG & Powell 1976 \\
\hline $\begin{array}{l}\text { powdered } \\
\text { leaves }\end{array}$ & boils & & China & Reynolds \& Fang 1940 \\
\hline $\begin{array}{l}\text { ash of } \\
\text { pseudostem, } \\
\text { corm, fruiting } \\
\text { stalk and } \\
\text { fruit peel }\end{array}$ & $\begin{array}{l}\text { anti-scorbutic, } \\
\text { digestive, tonic }\end{array}$ & edible cvs, $M$. ornata & N E India & Uma 2006, Watt 1972 \\
\hline \multicolumn{5}{|l|}{ Stimulant } \\
\hline dry leaves & $\begin{array}{l}\text { cigarette papers, } \\
\text { cigars }\end{array}$ & $\begin{array}{l}\text { many spp/cvs, } \\
\text { including Fe`i cvs, } M \text {. } \\
\text { campestris, } M \text {. hirta }\end{array}$ & widespread & $\begin{array}{l}\text { Christensen 2002, Heyne } \\
\text { 1950, Jacquat 1990, Lentfer } \\
\text { 2003, MacDaniels } 1947\end{array}$ \\
\hline \multicolumn{5}{|l|}{ Domestic Uses } \\
\hline \multirow[t]{2}{*}{ leaves } & wrapping material & $\begin{array}{l}\text { many spp/cvs, } \\
\text { including M. balbisiana, } \\
\text { M. maclayi, Musa } \\
\text { schizocarpa Simmonds }\end{array}$ & widespread & $\begin{array}{l}\text { Burkill 1935, Jacquat 1990, } \\
\text { Lentfer 2003, Nelson et al. } \\
\text { 2006, Powell 1976, Rajah } \\
\text { 2008, Simmonds 1959, } \\
\text { Sterly 1997, Watt } 1972\end{array}$ \\
\hline & $\begin{array}{l}\text { lining and covering } \\
\text { cooking vessels } \\
\text { and earth ovens }\end{array}$ & $\begin{array}{l}\text { many spp/cvs, including } \\
\text { M. ingens, M. peekelii }\end{array}$ & widespread & $\begin{array}{l}\text { Burkill 1935, Jacquat 1990, } \\
\text { Lentfer 2003, Nelson et al. } \\
\text { 2006, Powell 1976, Simmonds } \\
\text { 1959, Sterly 1997, Uma } 2006\end{array}$ \\
\hline $\begin{array}{l}\text { leaves, } \\
\text { midribs and } \\
\text { bracts }\end{array}$ & $\begin{array}{l}\text { plates, dishes } \\
\text { and mats }\end{array}$ & many spp/cvs & widespread & $\begin{array}{l}\text { Burkill 1935, Heyne 1950, } \\
\text { Jacquat 1990, MacDaniels 1947, } \\
\text { Nelson et al. 2006, Powell 1976, } \\
\text { Simmonds 1959, Sterly } 1997 \\
\end{array}$ \\
\hline \multirow[t]{2}{*}{ pseudostem } & raft, canoe rollers & Musa spp, Fe`i cvs & $\begin{array}{l}\text { N E India, } \\
\text { Micronesia, } \\
\text { PNG, } \\
\text { Polynesia }\end{array}$ & $\begin{array}{l}\text { MacDaniels 1947, Nelson } \\
\text { et al. 2006, Powell 1976, } \\
\text { Uma 2006, Williams } 1930\end{array}$ \\
\hline & $\begin{array}{l}\text { children's toy, } \\
\text { toboggan }\end{array}$ & edible Musa cvs & $\begin{array}{l}\text { PNG } \\
\text { Highlands }\end{array}$ & Sterly 1997 \\
\hline $\begin{array}{l}\text { outer skin of } \\
\text { pseudostem }\end{array}$ & containers & many spp/cvs & $\begin{array}{l}\text { PNG, } \\
\text { Mindanao }\end{array}$ & $\begin{array}{l}\text { Kocher Schmid 1991, Sterly } \\
\text { 1997, Yen \& Gutierrez } 1976\end{array}$ \\
\hline seedy fruit & beer additive & Eumusa spp & N E India & Uma 2006 \\
\hline $\begin{array}{l}\text { ash from } \\
\text { burnt } \\
\text { pseudostem, } \\
\text { leaves, corm }\end{array}$ & laundry/mordant & many spp/cvs & $\begin{array}{l}\text { India, } \\
\text { Malaysia, } \\
\text { Thailand }\end{array}$ & $\begin{array}{l}\text { Burkill 1935, Simmonds 1959, } \\
\text { Uma 2006, Watt } 1972\end{array}$ \\
\hline
\end{tabular}




\section{Kennedy - Bananas and People in the Homeland of Genus Musa: Not just pretty fruit}

\begin{tabular}{|c|c|c|c|c|}
\hline \multicolumn{5}{|l|}{ Category } \\
\hline Part used & Use & Genus/species & Location(s) & Citations \\
\hline juice of fruit & ink & & & Burkill 1935 \\
\hline \multirow[t]{2}{*}{$\begin{array}{l}\text { juice/sap from } \\
\text { pseudostem }\end{array}$} & $\begin{array}{l}\text { dye/stain for } \\
\text { bamboo/rattan, } \\
\text { matting }\end{array}$ & edible Musa cvs & $\begin{array}{l}\text { Indonesia, } \\
\text { Polynesia }\end{array}$ & $\begin{array}{l}\text { Heyne 1950, Nelson et al. } 2006 \text {, } \\
\text { Simmonds 1959, Uphof } 1968\end{array}$ \\
\hline & cleaning knives & edible Musa cvs & PNG & Kocher Schmid 1991 \\
\hline \multicolumn{5}{|l|}{ Shelter } \\
\hline \multirow[t]{3}{*}{ leaves } & $\begin{array}{l}\text { roofing and walls, } \\
\text { insulation and } \\
\text { draft-proofing }\end{array}$ & $\begin{array}{l}\text { many spp/cvs, including } \\
\text { M. ingens, } M \text {. schizocarpa }\end{array}$ & widespread & $\begin{array}{l}\text { Burkill 1935, Lentfer 2003, } \\
\text { Nelson et al. 2006, Powell } \\
\text { 1976, Simmonds 1959, } \\
\text { Sterly 1997, Watt } 1972\end{array}$ \\
\hline & temporary shelters & M. ingens & $\begin{array}{l}\text { PNG } \\
\text { Highlands }\end{array}$ & Lentfer 2003, Sterly 1997 \\
\hline & umbrella/raincoat & many spp/cvs & widespread & Burkill 1935, Simmonds 1959 \\
\hline petiole fiber & thatch & edible Musa cvs & Hawaii & Neal 1965 \\
\hline \multicolumn{5}{|l|}{ Cordage } \\
\hline $\begin{array}{l}\text { petiole and } \\
\text { pseudostem }\end{array}$ & $\begin{array}{l}\text { traditional cordage } \\
\text { and string }\end{array}$ & $\begin{array}{l}\text { many spp/cvs, including } \\
\text { Musa alinsanaya } \\
\text { R.V.Valmayor, } M . \\
\text { balbisiana, Musa textilis } \\
\text { Née, E. glaucum }\end{array}$ & widespread & $\begin{array}{l}\text { Burkill 1935, Heyne } 1950, \\
\text { Powell 1976, Rumphius } 1747 \text {, } \\
\text { Simmonds 1959, Sterly } 1997, \\
\text { Uphof 1968, Valmayor } 2001 \text {, Watt } \\
\text { 1972, Yen \& Gutierrez } 1976\end{array}$ \\
\hline pseudostem & industrial rope & M. textilis & Philippines & $\begin{array}{l}\text { Burkill 1935, Purseglove } \\
\text { 1975, Watt } 1972\end{array}$ \\
\hline \multirow[t]{2}{*}{ leaves } & rope & M. lasiocarpa & Yunnan & Liu et al. 2003 \\
\hline & string & Musa sp & Borneo & Christensen 2002 \\
\hline \multicolumn{5}{|l|}{ Clothing } \\
\hline leaves & $\begin{array}{l}\text { dried, shredded } \\
\text { for skirts, capes, } \\
\text { loin-cloths }\end{array}$ & many spp/cvs & $\begin{array}{l}\text { PNG, } \\
\text { Vanuatu }\end{array}$ & $\begin{array}{l}\text { Deacon 1934, Malinowski 1935, } \\
\text { Powell 1976, Weiner } 1976\end{array}$ \\
\hline pseudostem & $\begin{array}{l}\text { fiber for skirts, } \\
\text { skin for belts }\end{array}$ & many spp/cvs & Hawaii, PNG & Nelson et al. 2006, Powell 1976 \\
\hline \multicolumn{5}{|l|}{ Textiles } \\
\hline $\begin{array}{l}\text { pseudostem, } \\
\text { petiole }\end{array}$ & $\begin{array}{l}\text { fiber for cloth } \\
\text { production }\end{array}$ & $\begin{array}{l}\text { many spp/cvs, } \\
\text { especially } M . \text { textilis } \\
\text { and } M . \text { balbisiana }\end{array}$ & $\begin{array}{l}\text { Batanes, } \\
\text { Borneo, } \\
\text { S. China, } \\
\text { Luzon, } \\
\text { Micronesia, } \\
\text { Mindanao, } \\
\text { Ryukyus, } \\
\text { Sangihe/ } \\
\text { Talaud, } \\
\text { Solomon } \\
\text { Islands, } \\
\text { Vietnam, } \\
\text { Visayas }\end{array}$ & $\begin{array}{l}\text { Dampier 1927, Evans 1922, } \\
\text { Fraser-Lu 1988, Hendrickx } \\
\text { 2007, Kuhn 1988, Lu \& Huang } \\
\text { 1986, Reynolds \& Fang 1940, } \\
\text { Rubinstein \& Limol 2007, } \\
\text { Rumphius 1747, Stinchecum } 2007\end{array}$ \\
\hline \multirow{2}{*}{$\begin{array}{l}\text { juice/sap } \\
\text { from stalk of } \\
\text { inflorescence, } \\
\text { pseudostem } \\
\text { and suckers }\end{array}$} & $\begin{array}{l}\text { dye/stain for } \\
\text { yarn/cloth }\end{array}$ & edible Musa cvs & $\begin{array}{l}\text { India, } \\
\text { Indonesia, } \\
\text { Micronesia }\end{array}$ & $\begin{array}{l}\text { Heyne 1950, Rubinstein \& Limol } \\
\text { 2007, Rumphius 1747, Watt } 1972\end{array}$ \\
\hline & $\begin{array}{l}\text { dye/stain for } \\
\text { tapa cloth }\end{array}$ & Fe`i cvs & $\begin{array}{l}\text { PNG, } \\
\text { Samoa }\end{array}$ & Burkill 1935, Whistler 2000 \\
\hline
\end{tabular}




\begin{tabular}{|c|c|c|c|c|}
\hline \multicolumn{5}{|l|}{ Category } \\
\hline Part used & Use & Genus/species & Location(s) & Citations \\
\hline $\begin{array}{l}\text { pseudostem/ } \\
\text { petiole fiber, } \\
\text { skin of petiole }\end{array}$ & resist in tie-dying & edible Musa cvs & $\begin{array}{l}\text { E. } \\
\text { Indonesia, } \\
\text { Malaysia, } \\
\text { Ryukyus, } \\
\text { Thailand }\end{array}$ & $\begin{array}{l}\text { Burkill 1935, Fraser-Lu } \\
\text { 1988, Hendrickx } 2007\end{array}$ \\
\hline $\begin{array}{l}\text { skin of } \\
\text { pseudostem }\end{array}$ & $\begin{array}{l}\text { decorative element } \\
\text { in matting }\end{array}$ & Fe $i$ and other edible cvs & $\begin{array}{l}\text { Indonesia, } \\
\text { Polynesia }\end{array}$ & $\begin{array}{l}\text { Heyne 1950, MacDaniels } \\
\text { 1947, Neal 1965, Nelson et } \\
\text { al. 2006, Whistler } 2000\end{array}$ \\
\hline $\begin{array}{l}\text { ash from } \\
\text { burnt } \\
\text { pseudostem, } \\
\text { leaves, corm } \\
\end{array}$ & mordant & edible Musa cvs & India & Burkill 1935 \\
\hline $\begin{array}{l}\text { wax from } \\
\text { bract and leaf }\end{array}$ & $\begin{array}{l}\text { resist in batik } \\
\text { cloth production }\end{array}$ & M. acuminata ssp/var & Java & $\begin{array}{l}\text { Burkill 1935, Heyne } 1950, \\
\text { Nakai } 1948, \text { Simmonds } 1959\end{array}$ \\
\hline \multicolumn{5}{|l|}{ Paper-making } \\
\hline pseudostem & $\begin{array}{l}\text { fine and coarse } \\
\text { paper, industrial } \\
\text { filters, tea bags }\end{array}$ & many spp/cvs & widespread & $\begin{array}{l}\text { Burkill 1935, Hendrickx } 2007 \text {, } \\
\text { Sharrock 1996, Watt } 1972\end{array}$ \\
\hline $\begin{array}{l}\text { juice/sap } \\
\text { of corm }\end{array}$ & varnish for paper & M. balbisiana & Ryukyus & Hendrickx 2007 \\
\hline \multicolumn{5}{|l|}{ Ornamental } \\
\hline whole plant & horticultural & many spp/cvs & widespread & Constantine 1999-2008 \\
\hline inflorescence & horticultural & many spp/cvs & widespread & Constantine 1999-2008 \\
\hline flowers & garnish for food & M. peekelii & PNG & Lentfer 2003 \\
\hline leaves & body decoration & many spp/cvs & $\begin{array}{l}\text { PNG, } \\
\text { Polynesia }\end{array}$ & $\begin{array}{l}\text { Lentfer 2003, Powell } \\
\text { 1976, Whistler } 2000\end{array}$ \\
\hline dried leaves & garlands & many spp/cvs & widespread & Burkill 1935 \\
\hline seeds & $\begin{array}{l}\text { strung as beads, } \\
\text { ornaments }\end{array}$ & Musa and Ensete spp & $\begin{array}{l}\text { Philippines, } \\
\text { PNG, N. } \\
\text { Thailand }\end{array}$ & $\begin{array}{l}\text { Anderson 1993, Burkill 1935, } \\
\text { Lentfer 2003, Powell 1976, } \\
\text { Simmonds 1959, Williams } 1930\end{array}$ \\
\hline ash & tattooing & edible Musa cvs & Polynesia & Nelson et al. 2006 \\
\hline \multicolumn{5}{|c|}{ Ceremonial, Magic and Ritual } \\
\hline \multirow[t]{3}{*}{ whole plant } & $\begin{array}{l}\text { object of } \\
\text { veneration, symbol } \\
\text { of plenty, metaphor } \\
\text { for human } \\
\text { mortality, totem }\end{array}$ & edible Musa cvs & $\begin{array}{l}\text { India, } \\
\text { Madagascar, } \\
\text { Malaysia, } \\
\text { New } \\
\text { Caledonia, } \\
\text { PNG, } \\
\end{array}$ & $\begin{array}{l}\text { Endicott 1979, Kagy 1998, } \\
\text { Keller 2008, Watt 1972, } \\
\text { Williams 1936, Williams } 1941\end{array}$ \\
\hline & marker of graves & M. ingens & PNG & Argent 1979 \\
\hline & $\begin{array}{l}\text { many rituals, } \\
\text { exorcism, garden } \\
\text { magic, planted } \\
\text { as hunting decoy, } \\
\text { protective } \\
\end{array}$ & edible Musa cvs & $\begin{array}{l}\text { Malaysia, } \\
\text { PNG, } \\
\text { Polynesia }\end{array}$ & $\begin{array}{l}\text { Burkill 1935, Kocher Schmid 1991, } \\
\text { Malinowski 1935, Nelson et al. } \\
\text { 2006, Powell 1976, Sterly } 1997\end{array}$ \\
\hline fruiting stalk & ceremonial display & edible Musa cvs & $\begin{array}{l}\text { India, Bali, } \\
\text { Malaysia }\end{array}$ & Burkill 1935, Watt 1972 \\
\hline
\end{tabular}


Kennedy - Bananas and People in the Homeland of Genus Musa: Not just pretty fruit

\begin{tabular}{|c|c|c|c|c|}
\hline \multicolumn{5}{|l|}{ Category } \\
\hline Part used & Use & Genus/species & Location(s) & Citations \\
\hline \multirow[t]{2}{*}{ pseudostem } & $\begin{array}{l}\text { peace making } \\
\text { ceremony }\end{array}$ & edible Musa cvs & $\begin{array}{l}\text { PNG } \\
\text { Highlands }\end{array}$ & Sterly 1997 \\
\hline & funerary effigy & edible Musa cvs & $\begin{array}{l}\text { New Ireland, } \\
\text { PNG }\end{array}$ & Denner 2006, Peltier 2006 \\
\hline \multirow[t]{3}{*}{ leaves } & \begin{tabular}{|l} 
rituals, chewed \\
in funeral rites, \\
worn during dance \\
performance, \\
rain magic \\
\end{tabular} & edible Musa cvs & PNG & $\begin{array}{l}\text { Malinowski 1935, Powell } \\
\text { 1976, Sterly } 1997\end{array}$ \\
\hline & $\begin{array}{l}\text { ceremonies } \\
\text { and offerings }\end{array}$ & M. acuminata, M. hirta & $\begin{array}{l}\text { N. Thailand, } \\
\text { Borneo }\end{array}$ & $\begin{array}{l}\text { Anderson 1993, Christensen } \\
\text { 2002, Rajah } 2008\end{array}$ \\
\hline & $\begin{array}{l}\text { scraped, dried } \\
\text { bundles produced } \\
\text { and exchanged as } \\
\text { women's wealth }\end{array}$ & edible Musa cv & $\begin{array}{l}\text { Trobriand } \\
\text { Islands, } \\
\text { PNG }\end{array}$ & Weiner 1976 \\
\hline leaf sheath & divination & edible Musa cv & PNG & Kocher Schmid 1991 \\
\hline fruit & $\begin{array}{l}\text { offerings, in rites } \\
\text { of passage }\end{array}$ & edible Musa cvs & widespread & $\begin{array}{l}\text { Burkill 1935, Hla Pe 1978, } \\
\text { Kocher Schmid 1991, Rajah } \\
\text { 2008, Simmonds 1959, } \\
\text { Sterly 1997, Watt } 1972 \\
\end{array}$ \\
\hline stalk & surrogate human & edible Musa cvs & $\begin{array}{l}\text { Hawaii, } \\
\text { Tahiti }\end{array}$ & Neal 1965, Nelson et al. 2006 \\
\hline \multirow[t]{2}{*}{$\begin{array}{l}\text { juice/sap of } \\
\text { pseudostem }\end{array}$} & $\begin{array}{l}\text { cleansing ritual } \\
\text { for brides }\end{array}$ & edible Musa cvs & \begin{tabular}{|l} 
PNG \\
Highlands
\end{tabular} & Sterly 1997 \\
\hline & $\begin{array}{l}\text { paint/dye for } \\
\text { funerary masks }\end{array}$ & Fe`i cvs & PNG & Lentfer 2003 \\
\hline $\begin{array}{l}\text { skin of } \\
\text { pseudostem }\end{array}$ & $\begin{array}{l}\text { magic to strengthen } \\
\text { pigs and children }\end{array}$ & M. ingens & $\begin{array}{l}\text { PNG } \\
\text { Highlands }\end{array}$ & Sterly 1997 \\
\hline shoot & magic & M. ingens & $\begin{array}{l}\text { PNG } \\
\text { Highlands }\end{array}$ & Sterly 1997 \\
\hline
\end{tabular}

tions are unequivocally with the Indo-Malesian homeland. There is an extensive literature on African uses of Ensete (see Brandt et al. 1997, Constantine 1999-2008, Shigeta 1996, Tsehaye \& Kebebew 2006 for references).

Recognition of the usefulness of Musaceae was part of the basis of Sauer's (1952) hypothesis that agriculture in Southeast Asia began with the vegetative propagation of fiber and dye plants, followed by perennial food plants. Simmonds, accepting this part of Sauer's proposal, saw the recognition of the useful properties of translocated wild bananas by early Southeast Asians as the context in which 'the first signs of parthenocarpy and sterility [were seized upon] as providing a useful addition to an already considerable repertoire of virtues', during which 'the plants were transformed from jungle weeds into a highly productive crop' (Simmonds 1962:132).

The problem with this formulation is the shift of attention away from all useful aspects of Musaceae except edible fruit. The distortion is completely explicable, given that Simmonds' enormous contribution to cytogenetic research on banana edibility was aimed at the improvement of fruiting bananas. Nevertheless, it encourages ignorance, or amnesia, about other important aspects of the long history of entanglement of people and Musaceae. When Simmonds was considering such matters, the study of Southeast Asian prehistory had neither the tools to investigate past interrelationships of people and plants, nor any expectation of the great time depth involved.

The very widespread use of Musa species for fiber has certainly led in some places in directions that parallel those of fruiting bananas, with clonal reproduction of selected forms as well as planting from seed. In the Philippines, production of Musa textilis Née (Manila hemp, abacá), almost certainly a complex cultigen rather than a genuine wild species, was increased by Spanish decree in the mid-eighteenth century, and developed to industrial scale under later American and Japanese occupations, 
with production spreading to other countries (Hendrickx 2007, Purseglove 1975, Simmonds 1962). In the Philippines, production continues, despite the competition from synthetic fibers (Figure 6).

Production of other species for fiber has also been notable. For example, bashōfu cloth produced in Okinawa Prefecture was sent as tribute to China in the late sixteenth century, and is now a strong marker of a resurgent Okinawan identity (Figure 7). Popularized in Japan by a Japanese craft movement as an emblem of an idealized Okinawan past, the finest bashōfu cloth produced in Okinawa now commands high prices in mainland Japan - more than US $\$ 20,000$ for a kimono length. The plant is not Musa basjoo von Siebold ex Y. linuma as is commonly claimed (though that species does produce fiber), but Musa balbisiana Colla (Hendrickx 2007, Stinchecum 2007).

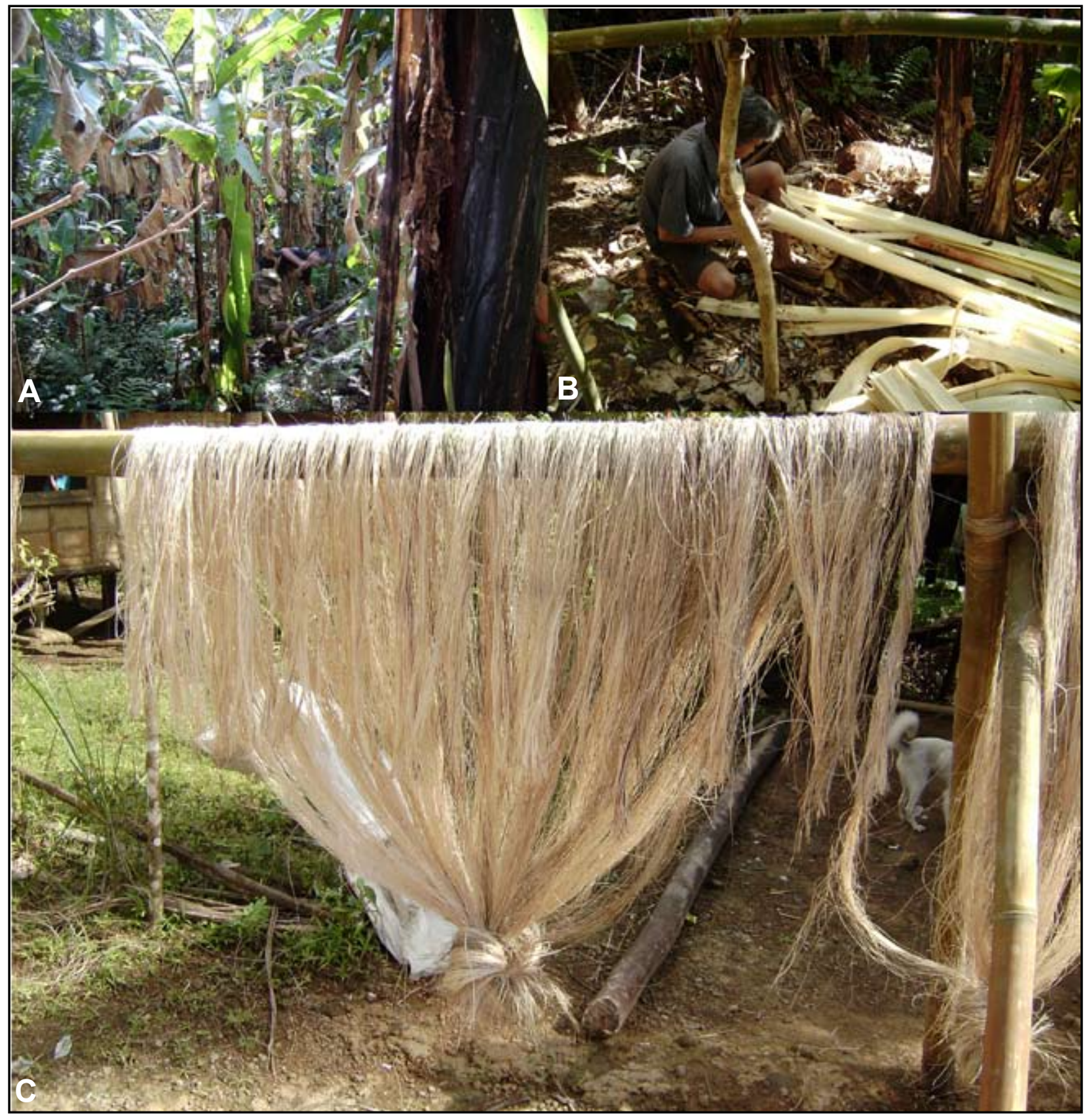

Figure 6. Production of abacá from Musa textilis Née, Aklan, Panay, Philippines: A. Harvesting in a cultivation area; B. Separating layers of pseudostem; C. Drying fiber. Photos by Juergen Steger, SachsenLeinen, Waldenburg, Germany. 


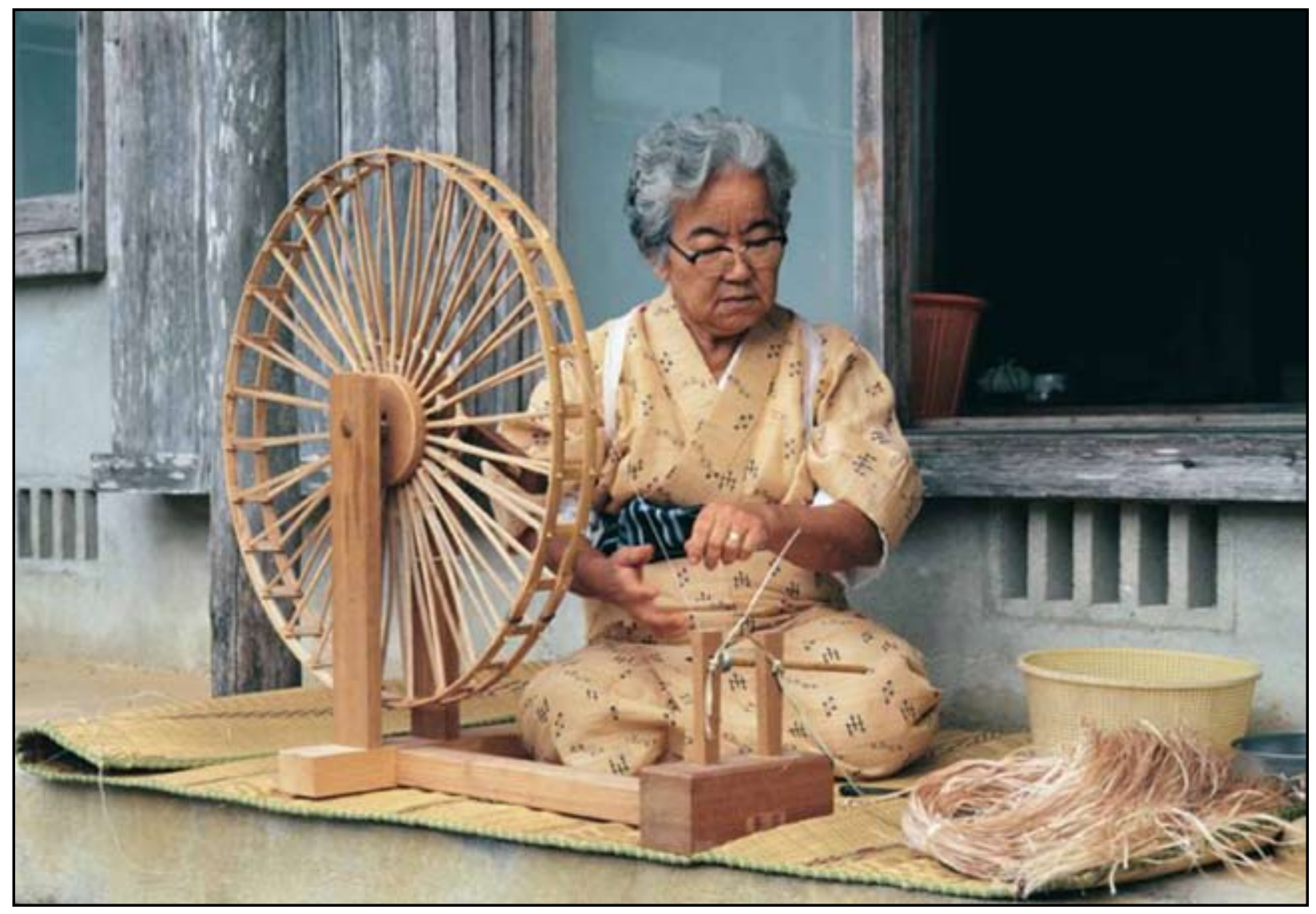

Figure 7. Winding thread of bashō fibre (from the inner pseudostem of Musa balbisiana Colla), the Okinawan weaver Yonemori Tokuko poses wearing a kimono of the same fibre, Maezato village, Ishigaki Island, Okinawa, 1983. Photo by Amanda Mayer Stinchecum, New York.

In the Trobriand Islands, Milne Bay Province, Papua New Guinea, women manufacture bundles and skirts from banana leaves by a laborious process involving scraping over carved wooden boards, producing distinctive decorative imprints, followed by sun-bleaching, drying and storage (K. Lepani pers. comm.) (Figure 8). These valuables are produced and exchanged only by women, and their labour in turning banana leaves into 'women's wealth' is central to women's control of the timeless cosmological domain which ensures the continuation of Trobriand social identity. Their role counterbalances the power of men in the quotidian world, to seek renown through the famous kula exchanges (Weiner 1976, 1980). The use of banana fiber in textile production is widespread in the Asia-Pacific region, and such textiles are often highly valued (Figure 9) (Fraser-Lu 1988, Hamilton 2007, Hendrickx 2007, Jolly 1992, Rubinstein \& Limol 2007, Weiner 1986).

\section{Conclusion}

These examples show that Indo-Malesian uses of Musaceae other than edible fruit are neither minor, nor necessarily secondary to production of the fruit. They have not dwindled into insignificance as the fruit became globally important. In the Philippines, abacá production, no longer important for rope, supports a burgeoning craft industry. In India, banana stems are processed for fiber and exported to Nepal, where knitting yarn is spun for sale in the West, marketed in craft shops as 'banana silk'. The cost of Okinawan bashōfu escalates. In the Trobriands, women continue to produce banana leaf bundles, and young women of Fais learn to weave machi.

Modern industries, whether on a huge scale, like the growing of Cavendish bananas for world trade, or the high value but small-scale production of bashōfu cloth in Okinawa, have grown from very deep roots. The industrial separation of fruit and fiber production should not blind us to the likelihood that particular Musa species in the past have furnished multiple products. Nevertheless, some specialization or differentiation is also likely, and this implies that selection may have taken different directions in different places and times. The example of the Ethiopian Ensete ventricosum (Welw.) Cheesman, domesticated as a starch staple, is well known (Brandt et al. 1997, Purseglove 1975). We should not rule out the possibility that 


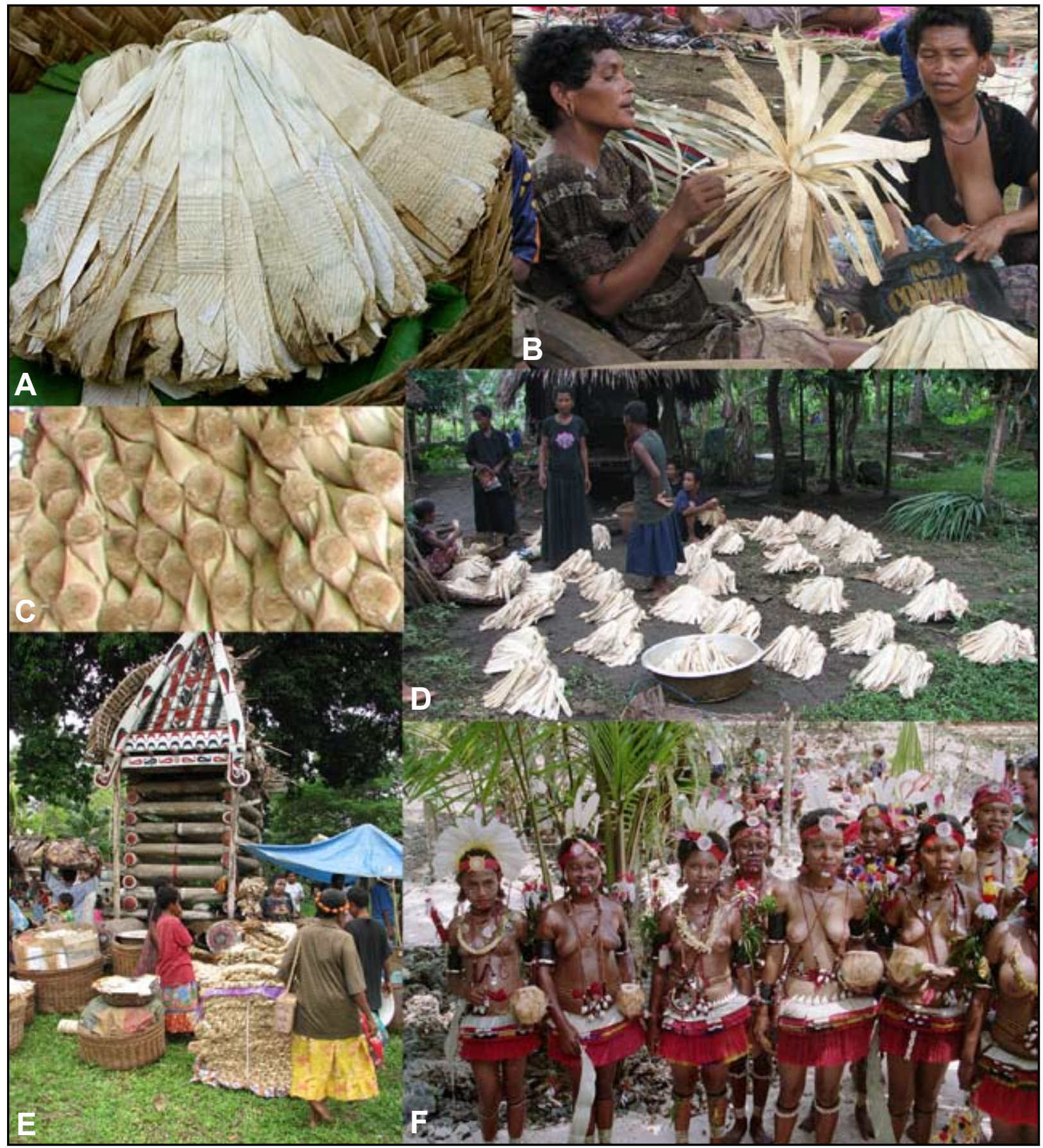

Figure 8. Banana leaf fiber is central to women's wealth (doba) in the Trobriand Islands, Milne Bay Province, PNG: A. The strips show decorative imprints; B. Tying prepared banana leaf strips into bundles; C. Close-up of piled bundles; $\mathbf{D}$. Preparing bundles in readiness for a ceremony; E. Piles of doba in front of a yam house during a ceremonial exchange; F. Young women's skirts are also produced from banana leaf strips. Photos by Mark Mosko, Anthropology Department, Research School of Pacific and Asian Studies, Australian National University.

parallel selection for starch production in the corm and pseudostem of Malesian Musa species may also have occurred in the past. There is at least one example of selection for enhanced starch storage in the rhizome of a
New Caledonian Musa plant, described as having a 'glaucous, violet stem and a turnip-like rhizome which, when cooked, resembles a yam in taste' (Simmonds 1959:267 citing Baker 1894). 


\section{Kennedy - Bananas and People in the Homeland of Genus Musa: Not just pretty fruit}

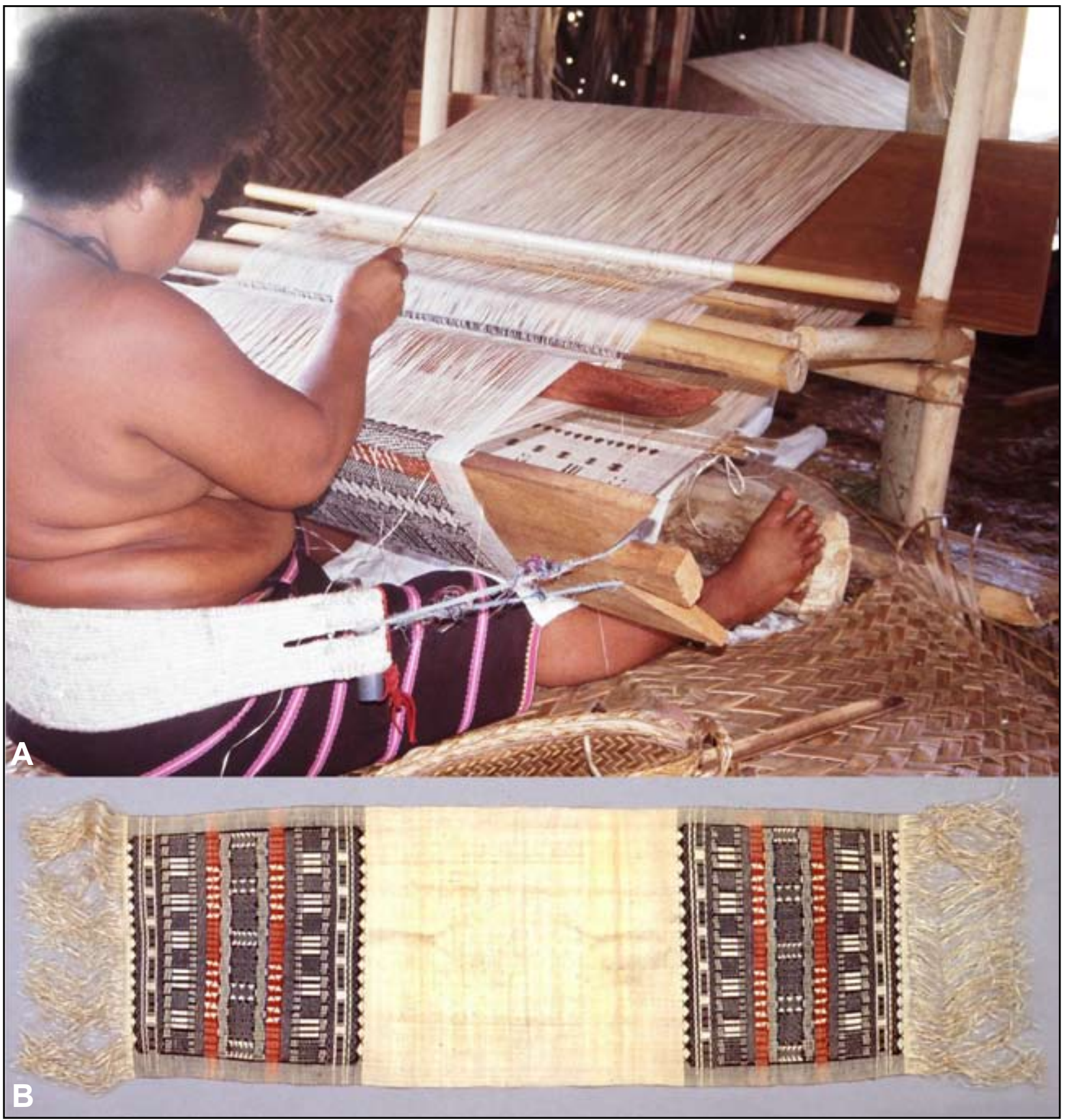

Figure 9. On Fais, in the western Caroline Islands of Micronesia, prestigious traditional textiles called machi are still woven. Fiber from Musa cultivars forms the warp and weft, with decorative supplementary weft of Hibiscus sp. fiber. The ritual value of machi derives from their association with chieftainship. A. Weaving on a back-strap loom; B. Machi. Photos by Michiko Intoh, National Museum of Ethnology, Osaka, Japan.

We should not, therefore, interpret archaeobotanical discoveries of banana parts by reference solely to what we know of the development of edibility in fruit. The cultivation of $M$. textilis and $M$. balbisiana for fiber show that seedy fruit is not necessarily an indication of 'wildness'. While parthenocarpy is the key to edibility of fruit, and its reproduction and transmission certainly depended upon vegetative propagation, other desirable characteristics of
Musa species may also have been vegetatively propagated. There is no reason to suppose that parthenocarpic fruit alone called forth propagation by vegetative planting. Although parthenocarpy could not have spread without the reinforcement of vegetative propagation, other desirable characteristics which did not interfere so directly with fertility could also be propagated in the same way. Thus, parthenocarpic forms of Musa species that on present evi- 
dence arose only on the eastern edge of Malesia may have spread west into areas where other useful Musa species were already well established in the domain of humans. The new fruit cultivars, and those that then developed from interspecific and intersubspecific hybridization, may thus have been an addition to the repertoire of banana cultivation, rather than an innovation or replacement.

\section{Acknowledgements}

Thanks to Tim Bayliss-Smith, Doreen Bowdery, Bill Clarke, David Constantine, Edmond De Langhe, Tim Denham, Peter Dwyer, Roy Ellen, James Fox, Jack Golson, Terry Hays, Robin Hide, Katherine Lepani, Peter Matthews, Doyle McKey, Mylien T. Nguyen, Rintaro Ono, Luc Vrydaghs, and Douglas Yen for their encouragement and assistance; and to Jeff Daniells, Michiko Intoh, Christiane Jacquat, Mark Mosko, Juergen Steger, Amanda Stinchecum and Shu Suehiro for their generosity in allowing me to reproduce their photographs.

\section{Literature Cited}

Anderson, E.F. 1993. Plants and People of the Golden Triangle: Ethnobotany of the hill tribes of Northern Thailand. Dioscorides Press, Portland, Oregon.

Argent, G. 1979. New Guinea bananas - a problematic genetic resource. Pp. 57-70 in Biological Aspects of Plant Genetic Resource Conservation in South-East Asia. Transactions of the Fifth Aberdeen-Hull Symposium on Malesian Ecology. Edited by K. Jong. Miscellaneous Series 21, Department of Geography, University of Hull, and Institute for South-East Asian Biology, University of Aberdeen.

Arnaud, E. \& J.-P. Horry. 1997. Editors of Musalogue: A catalogue of Musa germplasm. Papua New Guinea collecting expeditions, 1988-1989. International Network for the Improvement of Banana and Plantain, Montpellier.

Bailey, F.M. 1900. A New Guinea food plant. Queensland Agricultural Journal 7:442.

Baker, J.G. 1894. Species and principal varieties of Musa. Kew Bulletin 1894:119-314.

Barker, G. 2005. The archaeology of foraging and farming at Niah Cave, Sarawak. Asian Perspectives 44:90-106.

Barker, G., T. Reynolds \& D. Gilbertson. 2005. The human use of caves in peninsular and island Southeast Asia: Research themes. Asian Perspectives 44:1-15.

Barker G, H. Barton, M. Bird, P. Daly, I. Datan, A. Dykes, L. Farr, D. Gilbertson, B. Harrisson, C. Hunt, T. Higham, L. Kealhofer, J. Krigbaum, H. Lewis, S. McLaren, V. Paz, A. Pike, P. Piper, B. Pyatt, R. Rabett, T. Reynolds, J. Rose, G. Rushworth, M. Stephens, C. Stringer, J. Thompson \&
C. Turney. 2007. The 'human revolution' in lowland tropical Southeast Asia: The antiquity and behavior of anatomically modern humans at Niah Cave (Sarawak, Borneo). Journal of Human Evolution 52:243-261.

Brandt, S.A., A. Spring, C. Hiebsch, J.T. McCabe, E. Tabogie, M. Diro, G. Wolde-Michael, G. Yntiso, M. Shigeta \& S. Tesfaye. 1997. The 'Tree Against Hunger': Enset-based agricultural systems in Ethiopia. American Association for the Advancement of Science, Awassa Agricultural Research Center, Kyoto University Center for African Area Studies, and University of Florida, Washington, DC. www.aaas.org/ international/africa/enset/enset.pdf

Burkill, I.H. 1935. A Dictionary of the Economic Products of the Malay Peninsula. Crown Agents for the Colonies, London.

Carreel, F., D. González de Léon, P. Lagoda, C. Lanaud, C. Jenny, J.P. Horry \& H. Tézenas du Montcel. 2002. Ascertaining maternal and paternal lineage within Musa by chloroplast and mitochondrial DNA RFLP analyses. Genome 45:679-692.

Christensen, H. 2002. Ethnobotany of the Iban and Kelabit. Denmark and Sarawak, Malaysia, University of Aarhus, NEPCon and Forest Department, Sarawak.

Constantine, D. 1999-2008. The Musaceae: An annotated list of the species of Ensete, Musa and Musella. www.users.globalnet.co.uk/ drc/musaceae.htm. (Last accessed 20.1.2009).

Dampier, W. 1927. A New Voyage Round the World. Argonaut Press, London. (First published 1697.)

Dassanayake, M.D. \& W.D. Clayton. 2000. Editors of $A R e-$ vised Handbook to the Flora of Ceylon. Volume XIV. A.A. Balkema, Rotterdam.

Deacon, A.B. 1934. Malekula: A vanishing people in the New Hebrides. Routledge \& Sons, London.

Denner, A. 2006. Ephemeral art. Pp. 138-141 in New Ireland: Art of the South Pacific. Edited by M. Gunn \& P. Peltier. Musée du Quai Branly, Paris \& 5 Continents Editions, Milan.

De Langhe, E., L. Vrydaghs, P. de Maret, X. Perrier \& T. Denham. 2009. Why bananas matter: An introduction to the history of banana domestication. Ethnobotany Research and Applications 7:165-177.

De Langhe, E., D. Wattanachaiyingcharoen, H. Volkaert \& S. Piyapitchard. 2000. Biodiversity of wild Musaceae in Northern Thailand. Pp. 71-83 in Advancing Banana and Plantain $R$ and $D$ in Asia and the Pacific. Edited by A.B. Molina \& V.N. Roa. Proceedings of the 9th INIPAB-ASP- 


\section{Kennedy - Bananas and People in the Homeland of Genus Musa: Not just pretty fruit}

NET Regional Advisory Committee meeting. INIBAP-Asia and the Pacific Network, Los Baños, Laguna, Philippines.

Endicott, K.M. 1979. Batek Negrito Religion: The worldview and rituals of a hunting and gathering people of peninsular Malaysia. Clarendon Press, Oxford.

Evans, I.H.N. 1922. Among Primitive Peoples in Borneo. Seeley, Service, London.

Fraser-Lu, S. 1988. Handwoven Textiles of South-East Asia. Oxford University Press, Singapore, Oxford.

Groube, L. 1989. The taming of the rainforest: A model for late Pleistocene forest exploitation in New Guinea. Pp. 292-304 in Foraging and Farming: The evolution of plant exploitation. Edited by D. Harris \& G.C. Hillman. Unwin Hyman, London.

Häkkinen, M. 2006. Musa lawitiensis Nasution \& Supard. (Musaceae) and its intraspecific taxa in Borneo. Adansonia 28:55-65.

Häkkinen, M. \& H. Väre. 2008. Typification and check-list of Musa L. names (Musaceae) with nomenclatural notes. Adansonia série 3 30:63-112.

Häkkinen, M. \& H. Wang. 2007. New species and variety of Musa (Musaceae) from Yunnan, China. Novon 17:440446.

Häkkinen, M., H. Wang \& X.-J. Ge. 2008. Musa itinerans (Musaceae) and its intraspecific taxa in China. Novon 18:50-60.

Hamilton, R.W. 2007. Bast and leaf fibers in the Asia-Pacific region. Pp. 25-39 in Material Choices: Refashioning bast and leaf fibers in Asia and the Pacific. Edited by R.W. Hamilton \& B.L. Milgram. Fowler Museum, University of California at Los Angeles, Los Angeles.

Hays, T.E. 1991. Interest, use, and interest in uses in folk biology. Pp. 109-114 in Man and a Half: Essays in Pacific anthropology and ethnobiology in honour of Ralph Bulmer. Edited by A. Pawley. Memoir 48, The Polynesian Society, Auckland.

Hendrickx, K. 2007. The Origins of Banana-Fibre Cloth in the Ryukyus, Japan. Leuven University Press, Leuven.

Heyne, K. 1950. De Nuttige Planten van Indonesie. 3rd edition. N.V. Uitgeverij W. Van Hoeve, 's-Gravenhage, Bandung. (First published 1921.)

Hla Pe. 1978. Burmese attitudes to plants and animals. Pp. 88-104 in Natural Symbols in South East Asia. Edited by G.B. Milner. School of Oriental and African Studies, London.
Jacquat, C. 1990. Plants from the Markets of Thailand. Editions Duang Kamol, Bangkok, Thailand.

Jolly, M. 1992. Banana leaf bundles and skirts: A Pacific Penelope's web? Pp. 38-64 in History and Tradition in Melanesian Anthropology. Edited by J.G. Carrier. University of California Press, Berkeley, Los Angeles, Oxford.

Kagy, V. 1998. The food, socioeconomic and cultural importance of banana in Kanak society in New Caledonia. InfoMusa 7:22-24.

Kealhofer, L. 2003. Looking into the gap: Land use and the tropical forests of South Thailand. Asian Perspectives 42:72-95.

Keller, E. 2008. The banana plant and the moon: Conservation and the Malagasy ethos of life in Masoala, Madagascar. American Ethnologist 35:650-664.

Kennedy, J. 2008. Pacific bananas: Complex origins, multiple dispersals? Asian Perspectives 47:75-94.

Kennedy, J. n.d. Bananas: Towards a revised prehistory. in From Foragers to Farmers: Gordon C. Hillman festschrift. Edited by A.S. Fairbairn \& E. Weiss. Oxbow, Oxford. In press.

Kham, L. 2004. Medicinal Plants of Cambodia: Habitat, chemical constituents and ethnobotanical uses. Bendigo Scientific Press, Bendigo, Australia.

Kocher Schmid, C. 1991. Of People and Plants: A botanical ethnography of Nokopo village, Madang and Morobe Provinces, Papua New Guinea. Basler Beiträge zur Ethnologie, Band 33. Basel, Ethnologisches Seminar der Universität und Museum für Völkerkunde.

Kuhn, D. 1988. Textile Technology: Spinning and reeling. chemistry and chemical technology. Volume 5, Part 9. Science and Civilisation in China. Edited by J. Needham. Cambridge University Press, Cambridge.

Latinis, D.K. 2000. The development of subsistence system models for Island Southeast Asia and Near Oceania: The nature and role of arboriculture and arboreal-based economies. World Archaeology 32:41-67.

Lentfer, C.J. 2003. Tracing Antiquity of Banana Cultivation in Papua New Guinea: Report on collection of modern reference material from Papua New Guinea in 2002. Unpublished report prepared for the Pacific Biological Foundation, Sydney, Australia.

Liu, A.Z., W.J. Kress \& C.L. Long. 2003. The ethnobotany of Musella lasiocarpa (Musaceae), an endemic plant of southwest China. Economic Botany 57:279-281. 
Long, C., S. Ahmed, X. Wang, Y. Liu, B. Long, C. Yang, Y. Shi, X. Li \& R. Guo. 2008. Why Musella lasiocarpa (Musaceae) is used in southwest China to feed pigs. Economic Botany 62:182-186.

Lu, G.-D. \& H.-T. Huang. 1986. Botany, Biology and Biological Technology. Volume 6, Part 1. Science and Civilisation in China. Edited by J. Needham. Cambridge University Press, Cambridge.

MacDaniels, L.H. 1947. A Study of the Fe 'i Banana and its Distribution with reference to Polynesian Migrations. B.P. Bishop Museum Bulletin 190, Bishop Museum Press, Honolulu.

Malinowski, B. 1935. Coral Gardens and their Magic. American Book Company, New York, Cincinnati, Chicago.

Massal, E. \& J. Barrau, J. 1956. Food Plants of the South Seas. Technical Paper 94, South Pacific Commission, Noumea.

Mercader, J. 2003. Introduction: The Palaeolithic settlement of rain forests. Pp. 1-31 in Under the Canopy: The archaeology of tropical rain forests. Edited by J. Mercader. Rutgers University Press, New Brunswick, New Jersey.

Mogina, J. 2002. Changing Knowledge of Plants in Transitional Societies at Milne Bay, Papua New Guinea. Unpublished Ph.D. Thesis, Australian National University, Canberra.

Nakai, T. 1948. The kind of banana being wild or cultivated in west-Java, and their belongings. Bulletin of the Tokyo Science Museum 22:5-21

Neal, M.C. 1965. In Gardens of Hawaii. Bernice P. Bishop Museum Special Publication 50, Bishop Museum Press, Honolulu.

Nelson, S.C., R.C. Ploetz \& A.K. Kepler. 2006. Musa species (bananas and plantains), ver. 2.2. In Species Profiles for Pacific Island Agroforestry. Edited by C.R. Elevitch. Permanent Agriculture Resources (PAR), Hōlualoa, Hawaii i. www.traditionaltree.org.

Nguyen, M.T. 2006. Insertions and deletions: Evolution in the assemblage of Vietnamese food plants. Ethnobotany Research and Applications 4:175-201.

Noweg, T., A.R. Abdullah \& D. Nidang. 2003. Forest Plants as Vegetables for Communities Bordering the Crocker Range National Park. ASEAN Review of Biodiversity and Environmental Conservation (ARBEC). www.arbec.com. my/pdf/art3janmar03.pdf
Ochse, J.J. 1977. Vegetables of the Dutch East Indies. Australian National University Press, Canberra. (First published in Dutch, 1931.)

Pavlides, C. \& C. Gosden. 1994. 35,000-year-old sites in the rain forests of West New Britain, Papua New Guinea. Antiquity 68:604-610.

Peltier, P. 2006. Lihir Islands. Pp. 156-157 in New Ireland: Art of the South Pacific. Edited by M. Gunn \& P. Peltier. Musée du Quai Branly, Paris \& 5 Continents Editions, Milan.

Perrier X., F. Bakry, F. Carreel, Ch. Jenny, J.P. Horry, V. Lebot \& I. Hippolyte. 2009. Combining biological approaches to shed light on the evolution of edible bananas. Ethnobotany Research and Applications 7:199-216.

Perry, L.M. 1980. Medicinal Plants of East and Southeast Asia. MIT Press, Cambridge, Massachusetts.

Petir, A., D. Materem, P. Yaponmg, S. Mukarek, M. Okira \& T. Platts-Mills. 1998. Useful Plants of Salemben Village, Madang Province, Papua New Guinea. The Christensen Research Institute Publication No 13, Christensen Research Institute, Madang.

Powell, J.M. 1976. Ethnobotany. Pp. 106-183 in New Guinea Vegetation. Edited by K. Paijmans. Australian National University Press, Canberra.

Purseglove, J.W. 1975. Tropical Crops: Monocotyledons. 2nd impression. Halsted Press, New York.

Rajah, A. 2008. Remaining Karen: A study of cultural reproduction and the maintenance of identity. ANU E Press, Canberra.

Reynolds, P.K. \& C.Y. Fang. 1940. The banana in Chinese literature. Harvard Journal of Asiatic Studies 5:165-181.

Rubinstein, D. \& S. Limol. 2007. Reviving the sacred machi: A chiefly weaving from Fais Island, Micronesia. Pp. 155-165 in Material Choices: Refashioning bast and leaf fibers in Asia and the Pacific. Edited by R.W. Hamilton \& B.L. Milgram. Fowler Museum, UCLA, Los Angeles.

Rumphius, G.E. 1747. Herbarium Amboinense. Volume 5, Book 8. De plantis agens domesticis, tam victui, quam medicinae, et decori inservientibus. F. Changuion, H. Uytwerf, Amsterdam \& P. Gosse, J. Neaulme, A. Moetjens, A. van Dole, The Hague.

Sauer, C.O. 1952. Agricultural Origins and Dispersals: The domestication of animals and foodstuffs. American Geographical Society, New York. 


\section{Kennedy - Bananas and People in the Homeland of Genus Musa: Not just pretty fruit}

Sharrock, S. 1996. Uses of Musa. Focus Paper 3, INIBAP Annual Report. International Network for the Improvement of Banana and Plantain, Montpellier.

Shigeta, M. 1996. Creating landrace diversity: The case of the Ari people and enset (Ensete ventricosum) in Ethiopia. Pp. 233-268 in Redefining Nature: Ecology, culture and domestication. Edited by R. Ellen \& K. Fukui. Berg, Oxford.

Sillitoe, P. 1983. Roots of the Earth: Crops in the Highlands of Papua New Guinea. New South Wales Press, Kensington, Australia.

Simmonds, N.W. 1956. Botanical results of the banana collecting expedition, 1954-5. Kew Bulletin 11:463-489.

Simmonds, N.W. 1959. Bananas. Longmans, Green \& Co., London.

Simmonds, N.W. 1962. The Evolution of the Bananas. Longmans, Green \& Co., London.

Sterly, J. 1997. Simbu Plant Lore: Plants used by the people in the central highlands of New Guinea. Dietrich Reimer, Berlin.

Stinchecum, A.M. 2007. Bashōfu, the Mingei movement and the creation of a new Okinawa. Pp. 105-117 in Material Choices: Refashioning bast and leaf fibers in Asia and the Pacific. Edited by R.W. Hamilton \& B.L. Milgram. Fowler Museum, UCLA, Los Angeles.

Tsehaye, Y. \& F. Kebebew. 2006. Diversity and cultural use of enset (Ensete ventricosum (Welw.) Cheesman) in Bonga in situ conservation site, Ethiopia. Ethnobotany Research and Applications 4:147-157.

Uma, S. 2006. Farmers' Knowledge of Wild Musa in India. FAO, Rome.

Uphof, J.C.T. 1968. Dictionary of Economic Plants. J. Kramer, Lehre.

Valmayor, R.V. 2001. Classification and characterization of Musa exotica, $M$. alinsanaya and $M$. acuminata ssp. errans. Infomusa 10:35-39.

Watt, G. 1972. Dictionary of the Economic Products of India. Volume 5. Linum to Oyster. Periodical Experts, Delhi. (First published 1891.)
Weiner, A. 1976. Women of Value, Men of Renown: New perspectives in Trobriand exchange. University of Texas Press, Austin.

Weiner, A. 1980. Stability in banana leaves: Colonization and women in Kiriwina, Trobriand Islands. Pp. 270-293 in Women and Colonization: Anthropological perspectives. Edited by M. Etienne \& E. Leacock. J.F. Bergin, New York.

Weiner, A. 1986. Forgotten wealth: Cloth and women's production in the Pacific. Pp. 96-110 in Women's Work: Development and the division of labour by gender. Edited by E. Leacock, H.I. Safa. Bergin \& Garvey, Massachusetts.

Whistler, W.A. 1992. Polynesian Herbal Medicine. National Tropical Botanical Garden, Lawai, Kauai, Hawaii.

Whistler, W.A. 2000. Plants in Samoan Culture: The ethnobotany of Samoa. Isle Botanica, Hawaii.

Whitmore, T.C. 1984. Tropical Rain Forests of the Far East. Second edition, Oxford University Press, Oxford.

Williams, F.E. 1930. Orokaiva Society. Oxford University Press, London.

Williams, F.E. 1936. Papuans of the Trans-Fly. Oxford University Press, London.

Williams, F.E. 1941. Natives of Lake Kutubu, Papua. Oceania Monographs 6. Australian National Research Council, Sydney.

World Health Organisation (WHO) 1998. Medicinal Plants in the South Pacific. WHO, Manila.

Wu, D \& W.J. Kress. 2000. Musaceae. Pp. 297-313 in Flora of China. Volume 24 (Flagellariaceae through Marantaceae). Edited by Z.Y. Wu \& P.H. Raven. Science Press, Beijing, \& Missouri Botanical Garden Press, St. Louis.

Yen, D.E. \& H.G. Gutierrez. 1976. The ethnobotany of the Tasaday: I. The useful plants. Pp. 97-136 in Further Studies on the Tasaday. Edited by D.E. Yen \& J. Nance. Panamin Foundation Research Series 2, Makati, Philippines. 
